\title{
Fixed Point Theorems for Contractive Selfmappings of a Bounded Metric Space
}

\author{
Youssef Touail $\left(\mathbb{D},{ }^{1}\right.$ Driss El Moutawakil $\mathbb{D}^{\mathrm{D}},{ }^{1}$ and Samia Bennani ${ }^{2}$ \\ ${ }^{1}$ Equipe de Recherche en Mathématiques Appliquées, Technologies de l'Information et de la Communication, \\ Polydisciplinary Faculty of Khouribga, BP. 145, University Sultan Moulay Slimane, Morocco \\ ${ }^{2}$ Laboratory of Analysis, Algebra and Applications, Faculty of Sciences Ben M'sik BP. 7955, Sidi Othmane, \\ Universite Hassan II Casablanca, Casablanca, Morocco
}

Correspondence should be addressed to Youssef Touail; youssef9touail@gmail.com

Received 23 April 2019; Accepted 15 July 2019; Published 14 November 2019

Academic Editor: Pasquale Vetro

Copyright (C) 2019 Youssef Touail et al. This is an open access article distributed under the Creative Commons Attribution License, which permits unrestricted use, distribution, and reproduction in any medium, provided the original work is properly cited.

The main purpose of this paper is to prove a new fixed theorem for selfmapping of a metric space $(X, d)$. As applications, we get a new fixed point result for shrinking or contractive maps and a fixed point theorem for a new class of weakly contractive selfmappings of a bounded metric space $(X, d)$, where the auxiliary function $\phi$ satisfies $\phi(0)=0$ and $\inf _{t>0} \phi(t)>0$.

\section{Introduction}

Let $T: X \longrightarrow X$ be a mapping of a metric space $(X, d)$. It is well known that $T$ is called a shrinking or a contractive map if it satisfies the inequality $d(T x, T y)<d(x, y)$ for each $x, y \in X$ with $x \neq y$. In [1], V. V. Nemytzk was the first mathematician who studied the problem of the existence of a fixed point of these mappings. Furthermore, it is mentioned in [2] that, to obtain a fixed point of such mappings, it is necessary either to add the assumption that there exists a point $x \in X$ for which $\left\{T^{n} x\right\}$ contains a convergent subsequence, or else to assume that the space is compact.

In [3], the authors introduced the notion of weakly contractive mappings in Hilbert spaces and proved that any weakly contractive mapping defined on Hilbert spaces has a unique fixed point. Rhoades [4] proved that the corresponding result is also valid when Hilbert space is replaced by a complete metric space. Recall that a mapping $T: X \longrightarrow X$ of a metric space $(X, d)$ is said to be weakly contractive if $d(T x, T y) \leq d(x, y)-\phi(d(x, y))$, for all $x, y \in X$, where $\phi$ : $[0,+\infty[\longrightarrow[0,+\infty[$ is a continuous nondecreasing function such that $\phi(0)=0$. Since then, weakly contractive mappings have been dealt with in a number of papers. Some of these works are noted in $[1,5-8]$.
On the other hand, the authors [9] have introduced the concept of $\tau$-distance functions in a general topological space $(X, \tau)$ and mentioned that metric spaces, symmetric spaces, probabilistic metric spaces, and topological vector spaces have all such functions. Moreover, they presented an application of this new concept to the fixed point theory by giving the following result (Corollary 4.1 [9]) which generalizes the well-known Banach's fixed point theorem as follows

Theorem 1 (Corollary 4.1 [9]). Let $(X, \tau)$ be a Hausdorff topological space with a $\tau$-distance $p$. Suppose that $X$ is $p$ bounded and S-complete. Let $T: X \longrightarrow X$ be a mapping satisfying: there exists $k \in[0,1[$ such that for all $x, y \in X$, we have $p(T x, T y) \leq k p(x, y)$.

Then $T$ has a unique fixed point.

Recall that a sequence in $\mathrm{X}$ is p-Cauchy if it satisfies the usual metric condition with respect to $\mathrm{p}$. The definition of a p-bounded S-complete space is presented in Definition 3.1 [9] as follows.

Definition 2 (Definition 3.1 [9]). Let $(X, \tau)$ be a topological space with a $\tau$-distance $\mathrm{p}$. 
(1) $\mathrm{X}$ is S-complete if for every $\mathrm{p}$-Cauchy sequence $\left(x_{n}\right)$, there exists $\mathrm{X}$ in $\mathrm{X}$ with $\lim p\left(x, x_{n}\right)=0$.

(2) $\mathrm{X}$ is $\mathrm{p}$-Cauchy complete if for every p-Cauchy sequence $\left(x_{n}\right)$, there exists $\mathrm{x}$ in $\mathrm{X}$ with $\lim x_{n}=x$ with respect to $\tau$.

(3) $\mathrm{X}$ is said to be $\mathrm{p}$-bounded if $\sup \{p(x, y) / x, y \in X\}<$ $\infty$.

For more information, we refer the reader to [9]. Our purpose in this paper is to present a new fixed point result for shrinking maps and a fixed point theorem for a new class of weakly contractive selfmappings of a bounded metric space $(X, d)$ by using Theorem 1 , where the auxiliary function $\phi$ satisfies $\phi(0)=0$ and inf $t_{t>0} \phi(t)>0$.

\section{Main Results}

Theorem 3. Let $T: X \longrightarrow X$ be a mapping of a bounded complete metric space $(X, d)$ such that $\inf _{x \neq y \in X}\{d(x, y)-$ $d(T x, T y)\}>0$. Then $T$ has a unique fixed point.

Proof. Let $\alpha=\inf _{x \neq y \in X}\{d(x, y)-d(T x, T y)\}$. It is clear that, for all $x \neq y \in X$, one has $d(T x, T y) \leq d(x, y)-\alpha$, and, therefore $e^{d(T x, T y)} \leq k e^{d(x, y)}$, where $k=e^{-\alpha}$.

Let us consider the function $p: X \times X \rightarrow[0,+\infty[$ defined by

$$
p(x, y)= \begin{cases}e^{d(x, y)} & \text { if } x \neq y \\ 0 & \text { if } x=y\end{cases}
$$

As mentioned in [9] (Example 2.4.), the function $\mathrm{p}$ is a $\tau_{d^{-}}$ distance on $X$ where $\tau_{d}$ is the usual metric topology.

On the other hand, the mapping $T$ satisfies on $\left(X, \tau_{d}\right)$ the following contraction:

$$
\left.p(T x, T y) \leq k p(x, y), \quad \text { where } k=e^{-\alpha} \in\right] 0,1[\text {. }
$$

According to Corollary 4.1. in [9], we deduce that $T$ has a unique fixed point in $X$.

Corollary 4. Let $T: X \longrightarrow X$ be a shrinking mapping of a bounded complete metric space $(X, d)$ such that $\inf _{x \neq y \in X}\{d(x, y)-d(T x, T y)\} \neq 0$. Then $T$ has a unique fixed point.

Example 5. Let $X=\{1 ; 2\}$ with the usual metric $d(x, y)=$ $|x-y|$. Define $T$ by $T 1=1$ and $T 2=1$. We have

$$
d(1,2)-d(T 1, T 2)=1
$$

Then $T$ satisfies all assumptions of Corollary 4 and $T$ has the unique fixed point which is equal to 1 .

Example 6. Let $X=\bar{B}(0,1)$, the unit closed ball of a real Banach space, with the metric $d(x, y)=\left\{\begin{array}{ll}1 & \text { if } x \neq y \\ 0 & \text { if } x=y\end{array}\right.$.

Let us consider the mapping $T$ defined by $T x=0$, for all $x \in X$.
Then $T: X \longrightarrow X$ is a shrinking mapping of the complete bounded metric space $(X, d)$ and

$$
d(x, y)-d(T x, T y)=d(x, y)=1
$$

for all $x \neq y \in X$.

Therefore $T$ satisfies all assumptions of Corollary 4 and $T$ has the unique fixed point which is equal to 0 .

Remarks 7. Obviously, for a shrinking mapping $T: X \longrightarrow$ $X$ of a metric space $(X, d)$, one can ask does there exist a relationship between compactness and the condition $\inf _{x \neq y \in X}\{d(x, y)-d(T x, T y)\} \neq 0$. The answer is negative. Indeed, in the first example, the space $X$ is compact and $\inf _{x \neq y \in X}\{d(x, y)-d(T x, T y)\} \neq 0$. However, for $X=$ $[0,1]$ and $T x=(1 / 2) x$, the mapping $T$ is shrinking and $\inf _{x \neq y \in X}\{d(x, y)-d(T x, T y)\}=0$. Furthermore, in the second example, the space $(X, d)$ is not compact and $\inf _{x \neq y \in X}\{d(x, y)-d(T x, T y)\} \neq 0$.

Definition 8. Let $T: X \longrightarrow X$ be a mapping of a metric space $(X, d) . T$ will be said a E-weakly contractive maps if $d(T x, T y) \leq d(x, y)-\phi(d(x, y))$, for all $x, y \in X$, where $\phi:[0,+\infty[\longrightarrow[0,+\infty[$ is a function satisfying $\phi(0)=0$ and $\inf _{t>0} \phi(t)>0$.

As a second application of Theorem 3., we get the following new fixed point for E- weakly contractive selfmappings of a bounded metric space $(X, d)$.

Theorem 9. Let $T: X \longrightarrow X$ be a E-weakly contractive mapping of a bounded complete metric space $(X, d)$. Then $T$ has a unique fixed point.

Proof. From the Definition 8., it is clear that, for all $x \neq y \in$ $X$, we have

$$
0<\inf _{t>0} \phi(t) \leq \phi(d(x, y)) \leq d(x, y)-d(T x, T y),
$$

which implies that $\inf _{x \neq y \in X}\{d(x, y)-d(T x, T y)\}>0$. According to Theorem 3 . the mapping $T$ has a unique fixed point in $X$.

Example 10. Let $X=\{1 ; 2\}$ with the usual metric $d(x, y)=$

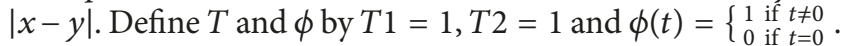

Then $T$ satisfies all assumptions of Theorem 9 and $T$ has the unique fixed point 1 . Note that $\phi$ is not continuous at 0 .

Example 11. Let $X=\{1 ; 2\}$ with the usual metric $d(x, y)=$ $|x-y|$. Define $T$ and $\phi$ by $T 1=2, T 2=1$, and $\phi(t)=0$ for all $t \in[0,+\infty[$.

Then $(X, d)$ is a bounded complete metric space and $d(T x, T y) \leq d(x, y)-\phi(d(x, y))$ for all $x, y \in X$. However, $T$ is not a E-weakly contractive since $\inf _{t>0} \phi(t)=0$ and $T$ has no fixed point. Therefore the condition that inf $f_{t>0} \phi(t) \neq 0$ is essential.

Example 12. Let $X=[0,+\infty[$ with the usual metric $d(x, y)=$ $|x-y|$. Define $T$ and $\phi$ by $T x=\ln \left(1+e^{x}\right)$ and $\phi(t)=t(1-$ 
$\sup \left\{T^{\prime}(x) \mid x \in[0,+\infty[\})\right.$ for all $x, t \in[0,+\infty[$, and $\mathrm{w}$ here $T^{\prime}$ is the derived function of $T$.

Then $T$ is a E-weakly contractive map with no fixed point on $X$ since $(X, d)$ is a complete unbounded metric space and $\inf _{t>0} \phi(t)=0$.

\section{Application}

In this section, we investigate the existence and uniqueness of a solution for the nonlinear integral equation:

$$
x(t)=f(t)+\int_{0}^{t} K(s, x(s)) d s,
$$

where $x \in \mathscr{C}[0, \tau]$ and the space of all continuous functions from $[0, \tau]$ into $\mathbb{R}$, with $\tau>0$.

$K:[0, \tau] \times \mathbb{R} \longrightarrow \mathbb{R}$ is a continuous mapping and $f:$ $[0, \tau] \longrightarrow \mathbb{R}$ is a given function.

Letting $X=\mathscr{C}[0, \tau]$ endowed by the metric $d: X \times X \longrightarrow$ $\mathbb{R}^{+}$defined by

$$
d(x, y)=\sup _{t \in[0, T]}|x(t)-y(t)|,
$$

obviously $(X, d)$ is a complete metric space.

Consider the mapping $T: X \longrightarrow X$ defined as follows:

$$
T(x)(t)=f(t)+\int_{0}^{t} K(s, x(s)) d s
$$

for any $x \in X$.

Note that (6) has a solution if and only if $T$ has a fixed point.

Under the above assumptions we have the following theorem.

Theorem 13. If there exists $M>0$ such that

$$
|K(s, x(s))-K(s, y(s))| \leq \frac{1}{\tau}[|x(s)-y(s)|-M],
$$

for all $s \in[0, \tau]$ and $x, y \in X$ such that $x \neq y$. Then the nonlinear integral equation (6) has a unique solution.

Proof. Assuming that $x, y \in X$ and $t \in[0, \tau]$, then we have

$$
\begin{aligned}
\mid T & (x)(t)-T(y)(t) \mid \\
& =\left|\int_{0}^{t} K(s, x(s)) d s-\int_{0}^{t} K(s, y(s)) d s\right| \\
& =\left|\int_{0}^{t}[K(s, x(s))-K(s, y(s))] d s\right| \\
& \leq \int_{0}^{t}|K(s, x(s))-K(s, y(s))| d s \leq d(x, y)-M
\end{aligned}
$$

hence

$$
d(T x, T y) \leq d(x, y)-M
$$

for all $x, y \in X$. Then $\inf _{x \neq y}\{d(x, y)-d(T x, T y)\} \geq M>$ 0 , which implies by Theorem 3 that there exists a unique solution of the nonlinear equation (6).

\section{Data Availability}

No data were used to support this study.

\section{Conflicts of Interest}

The authors declare that they have no conflicts of interest.

\section{References}

[1] V. V. Nemytzki, “The fixed point method in analysis Russian," Uspekhi Matematicheskikh Nauk, vol. 1, pp. 141-174, 1936.

[2] M. Edelstein, "On fixed and periodic points under contractive mappings," Journal of the London Mathematical Society, vol. 37, no. 1, pp. 74-79, 1962.

[3] Y. I. Alber and S. Guerre-Delabriere, "Principle of weakly contractive maps in Hilbert spaces," in New Results in Operator Theory and Its Applications, I. Gohberg and Y. Lyubich, Eds., vol. 98, pp. 7-22, Birkhäuser, Basel, Switzerland, 1997.

[4] B. E. Rhoades, "Some theorems on weakly contractive maps," Nonlinear Analysis-theory Methods \& Applications, vol. 47, no. 4, pp. 2683-2693, 2001.

[5] D. Doric, "Common fixed point for generalized $(\psi, \phi)$-weak contractions," Applied Mathematics Letters, vol. 22, no. 12, pp. 1896-1900, 2009.

[6] W. A. Kirk and N. Shahzad, Fixed Point Theory in Distance Spaces, Springer, Berlin, Germany, 2014.

[7] L. Ciric, Some Recent Results in Metrical Fixed Point Theory, University of Belgrade, Serbia, 2003.

[8] S. Radenović, Z. Kadelburg, D. Jandrlić, and A. Jandrlić, "Some results on weak contraction maps," Bulletin of the Iranian Mathematical Society, vol. 38, no. 3, pp. 625-645, 2012.

[9] M. Aamri and D. El Moutawakil, " $\tau$-distance in general topological spaces with application to fixed point theory," Southwest Journal of Pure and Applied Mathematics, no. 2, pp. 1-6, 2003. 


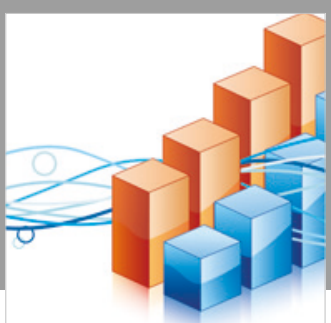

Advances in

Operations Research

\section{-n-m}
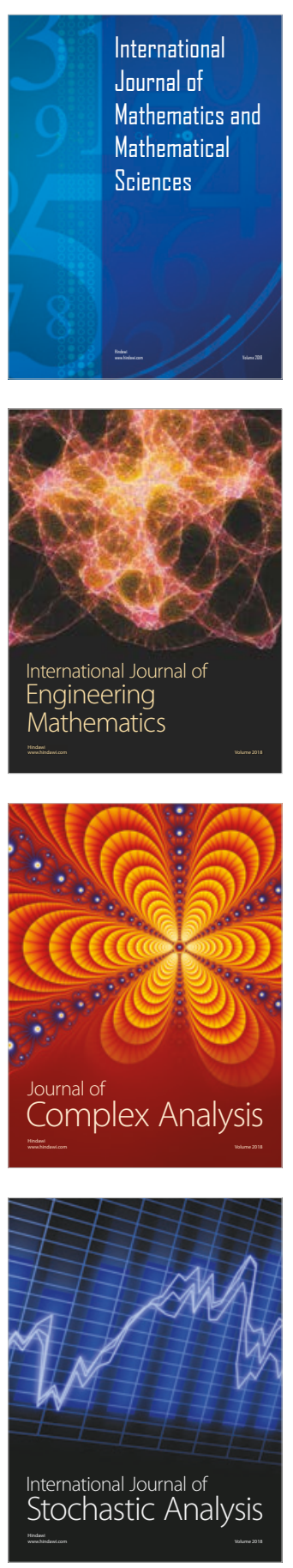
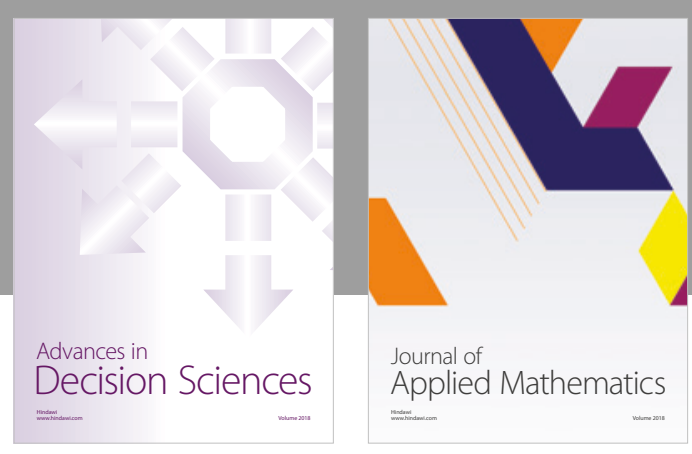

Journal of

Applied Mathematics
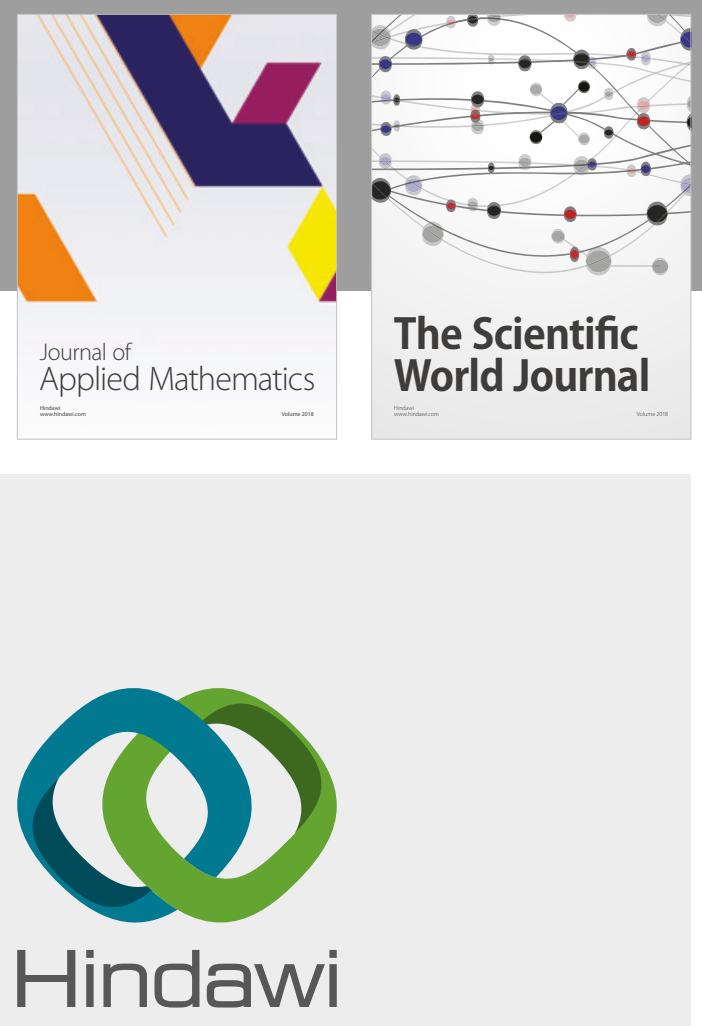

Submit your manuscripts at

www.hindawi.com

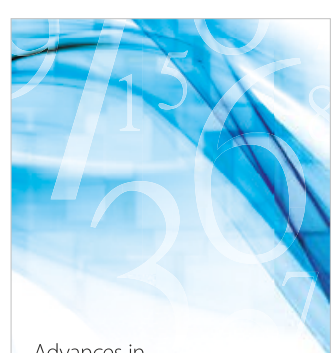

Advances in
Numerical Analysis
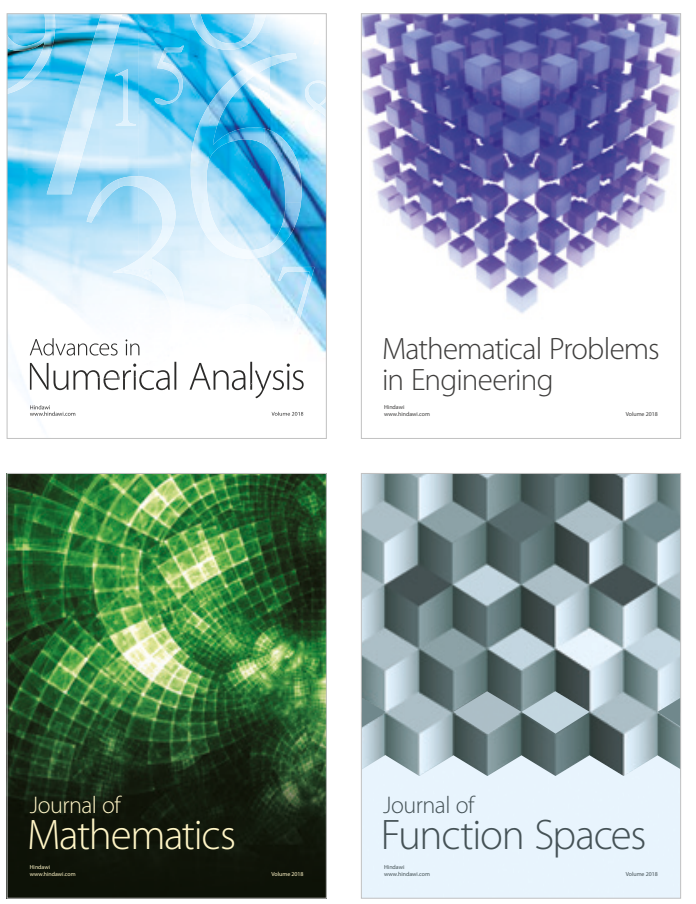

Mathematical Problems in Engineering

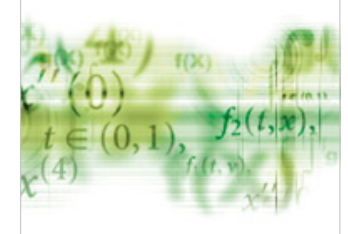

International Journal of

Differential Equations

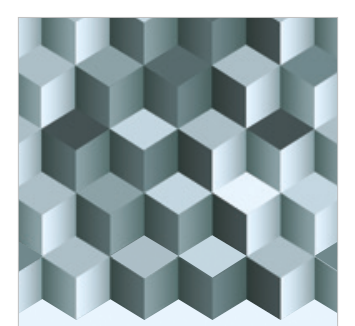

Journal of

Function Spaces

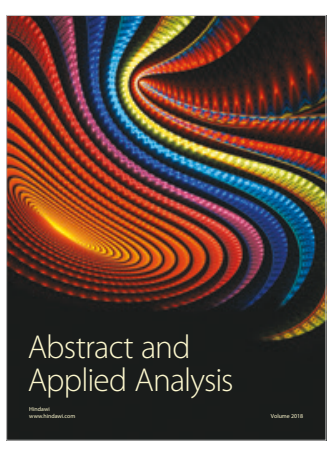

The Scientific

World Journal

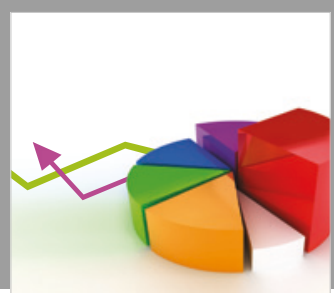

Journal of

Probability and Statistics
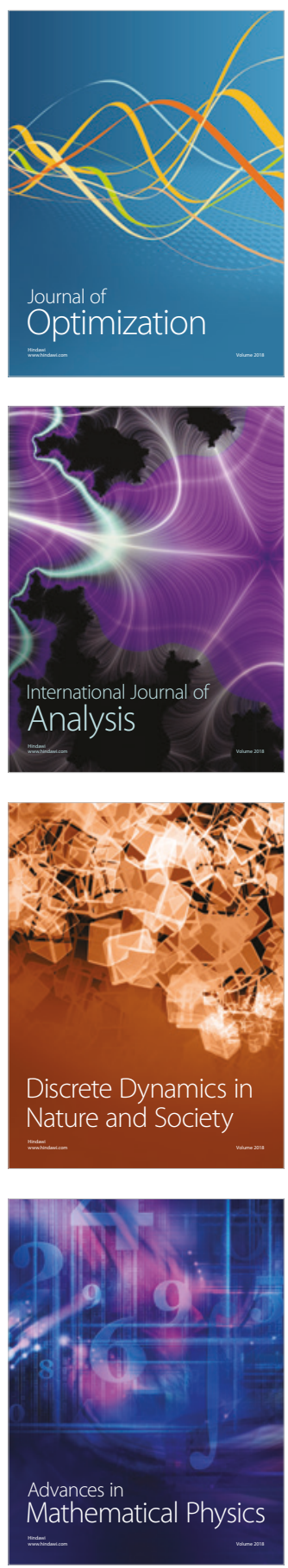